\title{
Homotopy perturbation method
}

Rajnee Tripathi and Hradyesh Kumar Mishra*

*Correspondence:

hk.mishra@juet.ac.in

Department

of Mathematics, Jaypee

University of Engineering

and Technology, Guna, MP

473226, India

\begin{abstract}
In this communication, we describe the Homotopy Perturbation Method with Laplace Transform (LT-HPM), which is used to solve the Lane-Emden type differential equations. It's very difficult to solve numerically the Lane-Emden types of the differential equation. Here we implemented this method for two linear homogeneous, two linear nonhomogeneous, and four nonlinear homogeneous Lane-Emden type differential equations and use their appropriate comparisons with exact solutions. In the current study, some examples are better than other existing methods with their nearer results in the form of power series. The Laplace transform used to accelerate the convergence of power series and the results are shown in the tables and graphs which have good agreement with the other existing method in the literature. The results show that LTHPM is very effective and easy to implement.
\end{abstract}

Keywords: Homotopy Perturbation Method (HPM), Laplace Transform (LT), Singular Initial value problems (IVPs), Lane-Emden type equations

\section{Background}

Two astrophysicists, Jonathan Homer Lane and Robert had explained the Lane-Emden type differential equations. In this study, they had designed these types of differential equations, which is a dimensionless structure of Poisson's equation for the gravitational potential of a self-gravitating, spherically symmetric, polytropic fluid and the thermal behavior of a spherical bunch of gas according to the laws of thermodynamics (Lane 1870; Richardson 1921). The Lane-Emden type of differential equation is also called the polytropic differential equations and it is given by:

$$
\frac{1}{\tau^{2}} \frac{d}{d \tau}\left(\tau^{2} \frac{d \Gamma}{d \tau}\right)+\Gamma^{n}=0
$$

where $\tau$ is a dimensionless radius and $\Gamma$ is linked to the density (and accordingly the pressure) by $\rho=\rho_{c} \tau^{n}$ for central density $\rho_{c}$. The index $\mathrm{n}$ is the polytropic index to make easy in the form of polytropic equation of state, $P=K \rho^{1+\frac{1}{n}}$, where $\mathrm{P}$ and $\rho$ are the pressure and $K$ density, respectively, and $\mathrm{n}$ is a constant of proportionality.

(c) The Author(s) 2016. This article is distributed under the terms of the Creative Commons Attribution 4.0 International License (http://creativecommons.org/licenses/by/4.0/), which permits unrestricted use, distribution, and reproduction in any medium, provided you give appropriate credit to the original author(s) and the source, provide a link to the Creative Commons license, and indicate if changes were made. 
Boundary conditions are

$$
\tau(0)=1, \quad \tau^{\prime}(0)=0 .
$$

Thus, the solutions describe the gallop of pressure and density (with radius), which is known as polytropes of $\mathrm{n}$.

The Lane-Emden equation has been useful to model some phenomena in astrophysics and mathematical physics such as the principle of stellar structure, the thermal nature of the spherical bunch of the gas, isothermal gas spheres (IGSs), and the principle of thermionic currents (Wazwaz 2011). According to the extensive study of many physicists, these equations have been applicable in the case of astrophysics such as kinetics of combustion and the Landau-Ginzburg major phenomenon (Dixon and Tuszynski 1990; Fermi 1927; Fowler 1930; Frank-Kamenetskii 1921). The numerical solutions of the Lane-Emden equations [LEes] are very difficult due to the expressive nature of the nonlinearities term. Therefore, much attention has been applied to the better and more powerful methods for establishing a solution, approximate or exact, analytical or numerical, to the Lane-Emden equations (LEes).

Recently many analytical techniques have been used for the solution of Lane-Emden type equation, for example, Hosseini and Abbasbandy (2015) described the hybrid Spectral Adomain Decomposition Method for solving Lane-Emden type of differential equations by combining the spectral method and Adomain Decomposition method. Since the description of this method is very long and difficult for solving these types of numerical problems. Our method is suitable and best to determine these results, Modified Laplace decomposition method for Lane-Emden type differential equations by Yin et al. (2013), A new algorithm for solving singular IVPs of Lane-Emden type differential equation by Motsa and Sibanda (2010). The author has solved the Lane-Emden type equations by Successive Linearization Method Since it is a very complicated method to get the solution of these types of problem in terms of an exact solution. Various methods for Lane-Emden equations have described by some authors in Rafiq et al. (2009), Baranwal et al. (2012), Liao (2003), Shawagfeh (1993), Wazwaz (2001), A new method for solving singular IVPs in the second order ordinary differential equations by Wazwaz (2001), Nouh (2004), Romas (2003) and other researchers have been studied several methods to attempt nonlinear problems. These methods have also been successfully applied to, analytical solution of convection-diffusion problem by combining Laplace transform method and homotopy perturbation method by Gupta et al. (2015), Mandelzweig and Quasi (2001), Singh et al. (2012), Nazari-Golshan et al. (2013), Explicit solution of Helmholtz equation and sixth-order KdV equation by Rafei and Ganji (2006), Ganji and Rajabi (2006), Jang (2016), Exact solutions of some coupled nonlinear partial differential equations(NPDE) using the homotopy perturbation method by Sweilam and Khader (2009), Homotopy perturbation method for solving viral dynamical model (VDM) by Merdan and Khaniyev (2010), nonlinear population dynamics models(NPDMs) by Chowdhury and Hashim (2007, Hashim and Chowdhury (2007), A new dispersion-relation preserving method for integrating the classical boussinesq equation by Jang (2017), The modified homotopy perturbation method (MHPM) for solving strongly nonlinear oscillators by Momani et al. (2009), Pandit (2014) and pure nonlinear differential by Cveticanin (2006), Inverse problem (IP) of diffusion equation 
by He's homotopy perturbation method by Shakeri and Dehghan (2007), A Higher order Numerical Scheme for singularly perturbed Burger-Huxley equation by Jiwari and Mittal (2011).

The Laplace transform is a superb technique for solving linear and nonlinear LaneEmden type differential equation and has enjoyed much success in the field of science and engineering. On the other hand, Laplace Transform (LT) has played an important role in mathematics (Spiegel and Teoríay 1988), not only for its theoretical interest but also because such method allows solving, in a simpler fashion, many problems in the realm of science, in comparison with other mathematical techniques. It is totally difficult to solve nonlinear equations because of the problems caused by nonlinear terms. Homotopy perturbation technique by He (1999), the homotopy perturbation method using Laplace Transform by Madani et al. (2011; Abbasbandy (2006); Gupta and Gupta 2011), a numerical solution of two-point boundary value problems using Galerkin-Finite element method by Sharma et al. (2012) have solved nonlinear problems. The Homotopy perturbation methods with Laplace transform (LT-HPM) and other methods have external significant thought in the literature. Moreover, The Homotopy Perturbation Method (HPM) by He (1999a, b, 2003) and the Variational Iteration Method (VIM) (Khuri and Sayfy 2012) are combined with the Laplace transform (LT) to develop a more effective technique for handling many nonlinear problems. A comparative study of model of matrix and finite elements methods for two-point boundary value problems is given by Sharma et al. (2012).

In the present paper, a Homotopy Perturbation Method (HPM) with Laplace Transform (LT) to solve the general type of Lane-Emden differential equations is proposed; the paper is organized as follows: The Homotopy Perturbation method is given in "Preliminaries" section. The Lane-Emden Equations (LEes) is given in "The Land-Emden equation" section. Homotopy Perturbation Method with Laplace Transform (LT-HPM) is given in "Homotopy perturbation method" section. Some examples of a different kind are given in "Results and discussion" section. Finally, the conclusion is explained in "Conclusions" section.

\section{Preliminaries}

\section{Homotopy perturbation method}

Consider the nonlinear differential equation

$$
A(\Gamma)-\zeta(r)=0, \quad r \in \Omega,
$$

with the boundary conditions of

$$
B\left(\Gamma, \frac{\partial \Gamma}{\partial n}\right)=0, \quad r \in \chi .
$$

where $\mathrm{A}, \mathrm{B}, \zeta(r)$ and $\chi$ are a general differential operator, a boundary operator, a known analytic function and the boundary of the domain $\Omega$, respectively and $\frac{\partial \Gamma}{\partial n}$ denotes the differentiation of $\Gamma$ with respect to $n$.

We can distribute the operator $\mathrm{A}$ into a linear part $\mathrm{K}$ and a nonlinear part N. Equation (1) may possibly be written as:

$$
K(\Gamma)+N(\Gamma)-\zeta(r)=0 .
$$


By the Homotopy technique, we construct a Homotopy $v(r, p): \Omega \times[0,1] \rightarrow R$ which satisfies:

$$
\begin{aligned}
& H(v, p)=(1-p)\left[K(v)-K\left(\Gamma_{0}\right)\right]+p[A(v)-\zeta(r)]=0, \\
& \text { or } \\
& H(v, p)=K(v)-K\left(\Gamma_{0}\right)+p K\left(\Gamma_{0}\right)+p[N(v)-\zeta(r)]=0,
\end{aligned}
$$

$p \in[0,1]$ is an embedding parameter, while $\Gamma_{0}$ is an initial approximation which satisfies the boundary conditions. Obviously, from above equations, we will have

$$
\begin{aligned}
& H(v, 0)=K(v)-K\left(\Gamma_{0}\right)=0 . \\
& H(v, 1)=A(v)-\zeta(r)=0 .
\end{aligned}
$$

Now changing the process of $p$ from 0 to 1 is even-handed that of $v(r, p)$ from $\Gamma_{0}$ to $\Gamma(r)$. According to the concept of topology, this is called deformation, whereas $K(v)-K\left(\Gamma_{0}\right)$ and $A(v)-\zeta(r)$ are called homotopy. If we consider the embedding parameter $p$ is a minor parameter, applying the classical perturbation technique, we can assume that the solution of Eqs. (4) and (5) can be defined as a power series in $p$ :

$$
v=v_{0}+v_{1} p+v_{2} p^{2}+v_{3} p^{3}+\cdots \cdots \infty
$$

putting $\mathrm{p}=1$ in Eq. (6), we have

$$
\Gamma=\lim _{p \rightarrow 1} v=v_{0}+v_{1}+v_{2}+v_{3}+\cdots \cdots
$$

The coupling of the perturbation method and the homotopy method is said to be HPM. The series (6) is convergent for most cases. However, the convergent rate depends on the nonlinear operator $A(v)$. Moreover, He (1999) made the following suggestions:

(1) The second derivative of $N(v)$ with respect to $v$ must be minor because the parameter may be comparatively large, i.e. $p \rightarrow 1$.

(2) The norm of $K^{-1}\left(\frac{\partial N}{\partial v}\right)$ must be lesser than one so that the series converges.

\section{Laplace transform method}

Definition The Laplace transform of a function $\xi(\tau)$, is defined by

$$
\xi(s)=L[\xi(\tau)]=\int_{0}^{\infty} e^{-s \tau} \xi(\tau) d \tau ; \quad \tau \geq 0
$$

(Whenever integral on RHS exists) where, $\tau \geq 0, \mathrm{~s}$ is real and $\mathrm{L}$ is the Laplace transform operator. 


\section{The Lane-Emden equation}

Lane-Emden type differential equations are singular initial value problems (IVPs) describing the second order homogeneous and nonhomogeneous linear and nonlinear differential equations which have been applicable in the many fields. The mathematical representation of Lane-Emden equation is:

$$
\Gamma^{\prime \prime}+\frac{2}{\tau} \Gamma^{\prime}+\zeta(\Gamma)=0, \quad 0 \leq \tau \leq 1,
$$

subject to conditions,

$$
\Gamma(0)=A, \quad \Gamma^{\prime}(0)=B .
$$

where A and B are constants $\zeta(\Gamma)$ is a real-valued continuous function.

These types of equations generally occur in the principle of stellar structure, the thermal behaviour of a spherical bunch of gas, isothermal gas spheres (IGSs) and the principle of thermionic currents (Richardson 1921; Chandrasekhar 1967; Davis 1962).

On the other hand, A nonlinear class of singular initial value problems of Lane-Emden type has the following form:

$$
\Gamma^{\prime \prime}+\frac{2}{\tau} \Gamma^{\prime}+\zeta(\tau, \Gamma)=\varphi(\tau), \quad 0 \leq \tau \leq 1
$$

The solution of the Lane-Emden type differential equation is numerically challenging because of the singularity behaviour at the origin. The solutions of the Lane-Emden equation were given by Wazwaz (2001), Shawagfeh (1993), Mishra (2014), the homotopy perturbation method (HPM) of above type by Davis (1962), Yildrim and Ozis (2007), He (2003), 2006), Ramos (2008), Exact solution of Generalized Lane-Emden equation is given by Goenner and Havas (2000). The major advantage of this method is its capability of combining the two powerful methods to obtain exact solutions of nonlinear equations. Therefore, Homotopy Perturbation Method using Laplace transform (LT-HPM) accelerates the rapid convergence of the series solution. In this paper, we will apply the (LT-HPM) to obtain exact or approximate analytical solutions of the Lane-Emden type equations.

\section{Homotopy perturbation method with laplace transform (LT-HPM)}

In this section, we will briefly discuss the use of the LT-HPM for the solution of LaneEmden equation given in "The Land-Emden equation" section, consider the following:

$$
\Gamma^{\prime \prime}+\frac{2}{\tau} \Gamma^{\prime}+\zeta(\tau, \Gamma)=\varphi(\tau), \quad 0 \leq \tau \leq 1 .
$$

Multiplying $\tau$ and then taking the Laplace transform on both sides of (11) we get:

$$
-s^{2} L^{\prime}(\Gamma)-\Gamma(0)+L\{\tau \zeta(\tau, \Gamma)-\tau \varphi(\tau)\}=0,
$$

where $L$ is the operator of Laplace transform and $L^{\prime}(\Gamma)=\frac{d L(\Gamma)}{d s}$

$$
L^{\prime}(\Gamma)=-s^{-2} \Gamma(0)+s^{-2} L[\tau \zeta(\tau, \Gamma)-\varphi(\tau)] .
$$

by integrating both sides of (13) with respect to s, we have 


$$
L(\Gamma)=-\int s^{-2} \Gamma(0) d s+\int s^{-2} L[\tau \zeta(\tau, \Gamma)-\tau \varphi(\tau)] d s
$$

taking inverse Laplace transform on both sides of (14), we get

$$
\Gamma(\tau)=-L^{-1}\left\{\int\left(s^{-2} \Gamma(0)\right) d s\right\}+L^{-1}\left\{\int s^{-2} L[\tau \zeta(\tau, \Gamma)-\tau \varphi(\tau)] d s\right\} .
$$

By using initial condition (9), we have

$$
\Gamma(\tau)=A+L^{-1}\left\{\int s^{-2} L[\tau \zeta(\tau, \Gamma)-\tau \varphi(\tau)] d s\right\} .
$$

We decompose $\zeta(\Gamma, \tau)$ into two parts

$$
\zeta(\tau, \Gamma)=K[\Gamma(\tau)]+N[\Gamma(\tau)] .
$$

where $K[\Gamma(\tau)]$ and $N[\Gamma(\tau)]$ denote the linear term and the nonlinear term respectively. The Homotopy perturbation method and the He's polynomials can be used to handle Eq. (12) and to address the nonlinear term. LT-HPM defines a solution by an infinite series of components given by:

$$
\Gamma(\tau)=\sum_{n=0}^{\infty} p^{n} \Gamma_{n}(\tau)
$$

where the terms $\Gamma_{n}(\tau)$ are to recursively calculate and the nonlinear term $\zeta(\Gamma)$ can be given as

$$
N(\Gamma)=\sum_{n=0}^{\infty} p^{n} H_{n}(\Gamma)
$$

where $N(\Gamma)$ is a non-linear term and $H_{n}(\Gamma)$ is He's polynomial.

For some He's polynomial $H_{n}$ (Mishra 2012) that are given by

$$
H_{n}\left(\Gamma_{0}, \Gamma_{1}, \Gamma_{2} \ldots \Gamma_{n}\right)=\frac{1}{n !} \frac{\partial^{n}}{\partial p^{n}}\left[N\left(\sum_{n=0}^{\infty} p^{i} \Gamma_{i}\right)\right]_{p=0} \quad n=0,1,2, \ldots
$$

Substituting the value of (19) and (20) in (18), we get

$$
\sum_{n=0}^{\infty} p^{n} \Gamma_{n}=A+p\left\{L^{-1} \int_{0}^{s} s^{-2}\left\{\left(L\left(\left(\sum_{n=0}^{\infty} p^{n} H_{n}(\tau)\right)-\tau\left(\sum_{n=0}^{\infty} p^{n} \Gamma_{n}(\Gamma)\right)\right)\right)\right\} d s\right\} .
$$

which is the coupling of the Laplace transformation and the Homotopy Perturbation Method (LT-HPM) using He's polynomials by Mishra $(2012,2014)$. Comparing the coefficient of like powers of $\mathrm{p}$, the following approximations are obtain 


$$
\left.\begin{array}{l}
p^{0}: \Gamma_{0}(x)=A, \\
p^{1}: \Gamma_{1}(\tau)=-L^{-1}\left[\int s^{-2}\left(\left(L\left(\tau\left(H_{0}\right)-\tau\left(\Gamma_{0}\right)\right)\right)\right) d s\right], \\
p^{2}: \Gamma_{2}(\tau)=-L^{-1}\left[\int s^{-2}\left(\left(L\left(\tau\left(H_{1}\right)-\tau\left(\Gamma_{1}\right)\right)\right)\right) d s\right], \\
p^{3}: \Gamma_{3}(\tau)=-L^{-1}\left[\int s^{-2}\left(\left(L\left(\tau\left(H_{2}\right)-\tau\left(\Gamma_{2}\right)\right)\right)\right) d s\right]
\end{array}\right\}
$$

\section{Results and discussion}

In this section, we will apply the method presented in this paper to solve singular IVPs of Lane-Emden-type.

Example 1 Consider the linear, homogeneous Lane-Emden differential equation

$$
\Gamma^{\prime \prime}+\frac{2}{\tau} \Gamma^{\prime}-2\left(2 \tau^{2}+3\right) \Gamma=0,
$$

with initial conditions

$$
\Gamma(0)=1 \quad \Gamma^{\prime}(0)=0 .
$$

Applying the Laplace transform on both sides, we get

$$
\begin{aligned}
L\left(\tau \Gamma^{\prime \prime}\right) & +2 L\left(\Gamma^{\prime}\right)-L\left(2\left(2 \tau^{3}+3 \tau\right) \Gamma\right)=0, \\
& -s^{2} L^{\prime}(\Gamma)-1=L\left\{2\left(2 \tau^{3}+3 \tau\right) \Gamma\right\}, \\
L^{\prime}(\Gamma)= & -\frac{1}{s^{2}}-\frac{L}{s^{2}}\left\{2\left(2 \tau^{3}+3 \tau\right) \Gamma\right\} .
\end{aligned}
$$

By integrating both sides of Eq. (25) with respect to s, we have

$$
\Gamma(s)=\int L(\Gamma) d s=-\int s^{-2} d s-\int s^{-2} L\left(2\left(2 \tau^{3}+3 \tau\right) \Gamma\right) d s .
$$

Taking Inverse Laplace transform on both sides, we get

$$
\begin{aligned}
L^{-1}(\Gamma(s)) & =-L^{-1}\left(\int s^{-2} d s\right)-\left(\int s^{-2} L\left(2\left(2 \tau^{3}+3 \tau\right) \Gamma\right) d s\right), \\
\Gamma(\tau) & =-L^{-1}\left(\int s^{-2} d s\right)-\left(\int s^{-2} L\left(2\left(2 \tau^{3}+3 \tau\right) \Gamma\right) d s\right), \\
\Gamma(\tau) & =1-L^{-1}\left(\int \frac{L}{s^{2}}\left(2\left(2 \tau^{3}+3 \tau\right) \Gamma\right) d s\right) .
\end{aligned}
$$

Applying Homotopy perturbation method, we get a solution by an infinite series of components given by:

$$
\Gamma(\tau)=\sum_{n=0}^{\infty} p^{n} \Gamma_{n}(\tau) .
$$

Thus Eq. (23) becomes 


$$
\sum_{n=0}^{\infty} p^{n} \Gamma_{n}(\tau)=1-p L^{-1}\left(\int s^{-2}\left(L\left(\sum_{n=0}^{\infty} p^{n}\left(2\left(2 \tau^{3}+3 \tau\right) \Gamma_{n}(\tau)\right) d s\right)\right) .\right.
$$

Equating the coefficient of like power of $\mathrm{p}$, we get

$$
\begin{aligned}
& p^{0}: \Gamma_{0}=1 \\
& p^{1}: \Gamma_{1}=\tau^{2}+\frac{\tau^{4}}{5} \\
& p^{2}: \Gamma_{2}=\frac{3}{10} \tau^{4}+\frac{13}{105} \tau^{6}+\frac{1}{90} \tau^{8} \\
& p^{3}: \Gamma_{3}=\frac{3}{70} \tau^{6}+\frac{17}{630} \tau^{8}+\frac{59}{11550} \tau^{10}+\frac{\tau^{12}}{3510}, \\
& p^{4}: \Gamma_{4}:=\frac{\tau^{8}}{280}+\frac{\tau^{10}}{330}+\frac{343 \tau^{12}}{386100}+\frac{4987 \tau^{14}}{47297250}+\frac{\tau^{16}}{238680},
\end{aligned}
$$

Table 1 The comparison with exact solution and ADM (Wazwaz 2001)

\begin{tabular}{llllll}
\hline $\boldsymbol{\tau}$ & LT-HPM & ADM & Exact & Error (LT-HPM) & Error (ADM) \\
\hline 0.0 & 1 & 1 & 1 & 0.0 & 0.0 \\
0.1 & 1.0100501670842 & 1.01005017 & 1.0100501670842 & 0.0 & 0.0 \\
0.2 & 1.0408107741924 & 1.04081078 & 1.0408107741924 & 0.0 & $1 \mathrm{E}-9$ \\
0.3 & 1.0941742836956 & 1.09417428 & 1.0941742837052 & $9.6 \mathrm{E}-12$ & $1 \mathrm{E}-9$ \\
0.4 & 1.1735108704484 & 1.17351087 & 1.1735108709918 & $5.434 \mathrm{E}-10$ & $2.3 \mathrm{E}-8$ \\
0.5 & 1.2840254041884 & 1.28402542 & 1.2840254166877 & $1.24993 \mathrm{E}-8$ & $3.5 \mathrm{E}-7$ \\
0.6 & 1.4333292517888 & 1.43332623 & 1.4333294145603 & $1.627715 \mathrm{E}-7$ & $3.2 \mathrm{E}-6$ \\
0.7 & 1.6323147871342 & 1.63229556 & 1.6323162199554 & $1.4328212 \mathrm{E}-6$ & $2.1 \mathrm{E}-5$ \\
0.8 & 1.8964714019044 & 1.89637596 & 1.8964808793049 & $9.99706 \mathrm{E}-4$ & $1.1 \mathrm{E}-4$ \\
\hline & & & & & \\
\hline
\end{tabular}

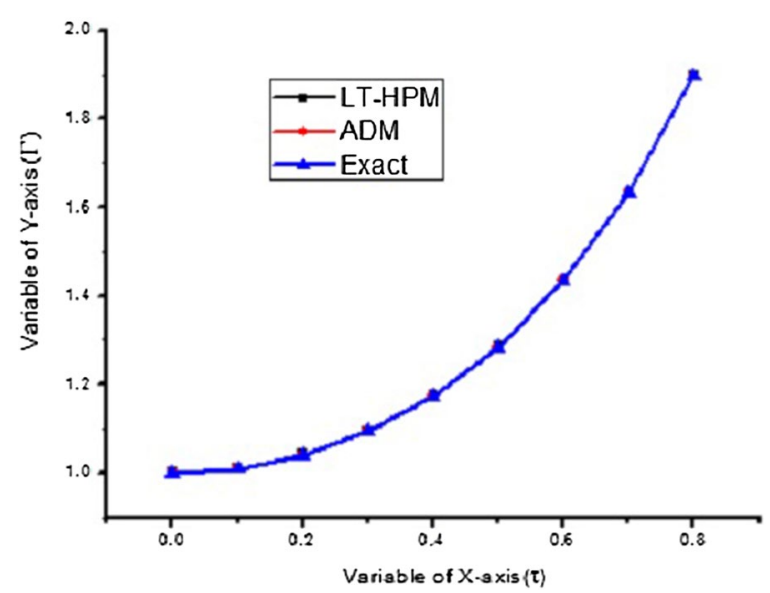

Fig. 1 Comparison between LT-HPM, Exact and ADM solution 
Thus we get the solution of this series as follows:

$$
\begin{aligned}
\Gamma & =\Gamma_{0}+\Gamma_{1}+\Gamma_{2}+\Gamma_{3}+\Gamma_{4}+\cdots, \\
& =1+\tau^{2}+\frac{\tau^{4}}{2}+\frac{\tau^{6}}{6}+\frac{\tau^{8}}{24}+\frac{\tau^{10}}{120}+\frac{\tau^{12}}{720} \cdots .
\end{aligned}
$$

The closed form of the series (28) is $y=\exp \left(\tau^{2}\right)$ which gives an approximate solution of the problem.

In this problem, the Table 1 shows the comparison of values of LT-HPM with exact and ADM in the terms of different values of $\tau$ (Fig. 1).

The graphical comparison of LT-HPM, exact solution and ADM solution are given as follows:

Example 2 Consider the linear, homogeneous Lane-Emden differential equation

$$
\Gamma^{\prime \prime}+\frac{2}{\tau} \Gamma^{\prime}+\Gamma^{n}=0, \quad \tau \geq 0, \quad n=0,1, \ldots
$$

subject to the initial condition

$$
\Gamma(0)=1, \quad \Gamma^{\prime}(0)=0 .
$$

Taking Laplace transform on both sides, we get

$$
\begin{aligned}
& L\left(\tau \Gamma^{\prime \prime}\right)+2 L\left(\Gamma^{\prime}\right)+L\left(\tau \Gamma^{n}\right)=0, \\
& \quad-s^{2} L^{\prime}(\Gamma)-\Gamma(0)+L\left(\tau \Gamma^{n}\right)=0,
\end{aligned}
$$

putting $\mathrm{n}=0$ in Eq. (31), we get

$$
\begin{aligned}
& -s^{2} L^{\prime}(\Gamma)-\Gamma(0)+L(\tau)=0 . \\
& L^{\prime}(\tau)=-\frac{1}{s^{2}}+\frac{1}{s^{4}} .
\end{aligned}
$$

Integrating above equation with respect to $\mathrm{s}$, we get

$$
L(\tau)=\int\left(-s^{-2}+s^{-4}\right) d s .
$$

Taking inverse Laplace Transform on both sides, we get

$$
\begin{aligned}
& \Gamma(\tau)=L^{-1} \int\left(-\frac{1}{s^{2}}+\frac{1}{s^{4}}\right) d s, \\
& \Gamma(\tau)=1-\frac{\tau^{2}}{6} .
\end{aligned}
$$

which is the exact solution.

When $\mathrm{n}=1$ in Eq. (31), we get

$$
\begin{aligned}
& -s^{2} L^{\prime}(\Gamma)-\Gamma(0)+L(\tau \Gamma)=0 . \\
& L^{\prime}(\Gamma)=-\frac{1}{s^{2}}+\frac{1}{s^{2}} L(\tau \Gamma)=0
\end{aligned}
$$


Integrating above equation with respect to $s$, we get

$$
L(\Gamma(\tau))=-\int s^{-2} d s+\int s^{-2} L(\tau \Gamma) d s
$$

Taking inverse Laplace transform on both sides, we get

$$
\begin{aligned}
& \Gamma(\tau)=-L^{-1}\left(\int s^{-2} d s\right)+L^{-1}\left(\int s^{-2} L(\tau \Gamma) d s\right), \\
& \Gamma(\tau)=1+L^{-1}\left(\int s^{-2} L(\tau \Gamma) d s\right) .
\end{aligned}
$$

Applying Homotopy Perturbation Method on both sides, we get

$$
\sum_{n=0}^{\infty} p^{n} \Gamma_{n}(\tau)=1+p L^{-1}\left(\int s^{-2}\left(\sum_{n=0}^{\infty} p^{n} \Gamma_{n}(\tau)\right) d s\right) .
$$

Equating the coefficient of like power of $\mathrm{p}$, we get

$$
\begin{aligned}
& p^{0}: \Gamma_{0}=1 \\
& p^{1}: \Gamma_{1}=-L^{-1}\left(\int s^{-2} L\left(\Gamma_{0}\right) d s\right)=-\frac{\tau^{2}}{6} \\
& p^{2}: \Gamma_{2}=-L^{-1}\left(\int s^{-2} L\left(\Gamma_{1}\right) d s\right)=\frac{\tau^{4}}{5 \times 4 !} \\
& p^{3}: \Gamma_{3}=-L^{-1}\left(\int s^{-2} L\left(\Gamma_{2}\right) d s\right)=-\frac{\tau^{6}}{7 \times 6 !} \\
& p^{4}: \Gamma_{4}=-L^{-1}\left(\int s^{-2} L\left(\Gamma_{3}\right) d s\right)=\frac{\tau^{8}}{9 \times 8 !},
\end{aligned}
$$

Thus we get the solution of this series as follows:

$$
\begin{aligned}
\Gamma & =\Gamma_{0}+\Gamma_{1}+\Gamma_{2}+\Gamma_{3}+\Gamma_{4}+\cdots \\
& =1-\frac{\tau^{2}}{6}+\frac{\tau^{4}}{5 \times 4 !}-\frac{\tau^{6}}{7 \times 6 !}+\frac{\tau^{8}}{9 \times 8 !}+\cdots .
\end{aligned}
$$

The closed form of the series (32) is $\Gamma(\tau)=\frac{\sin \tau}{\tau}$ which gives an exact solution of the problem.

The Table 2 shows the comparison of values of LT-HPM within the terms of different values of $\tau$.

Table 2 Comparison with exact solution

\begin{tabular}{llll}
\hline $\boldsymbol{\tau}$ & LT-HPM & Exact & Error (LT-HPM) \\
\hline 0.1 & 0.9983341665 & 0.998334166 & $-5 \mathrm{E}-10$ \\
0.2 & 0.993346654 & 0.993346654 & $-0 \mathrm{E}+0$ \\
0.3 & 0.9850673555 & 0.9850673555 & $-0 \mathrm{E}+0$ \\
0.4 & 0.9735458558 & 0.9735458558 & $-0 \mathrm{E}+0$ \\
0.5 & 0.9588510772 & 0.9588510772 & $-0 \mathrm{E}+0$ \\
0.6 & 0.9410707891 & 0.941070789 & $-1 \mathrm{E}-10$ \\
0.7 & 0.9203109825 & 0.9203109818 & $-7 \mathrm{E}-10$ \\
0.8 & 0.8966951163 & 0.8966951136 & $-2.7 \mathrm{E}-9$ \\
0.9 & 0.8703632416 & 0.8703632329 & $-8.7 \mathrm{E}-9$ \\
1.0 & 0.8414710097 & 0.8414709848 & $-2.49 \mathrm{E}-8$ \\
\hline
\end{tabular}


We established the graphical comparison of LT-HPM and exact solution as follows:

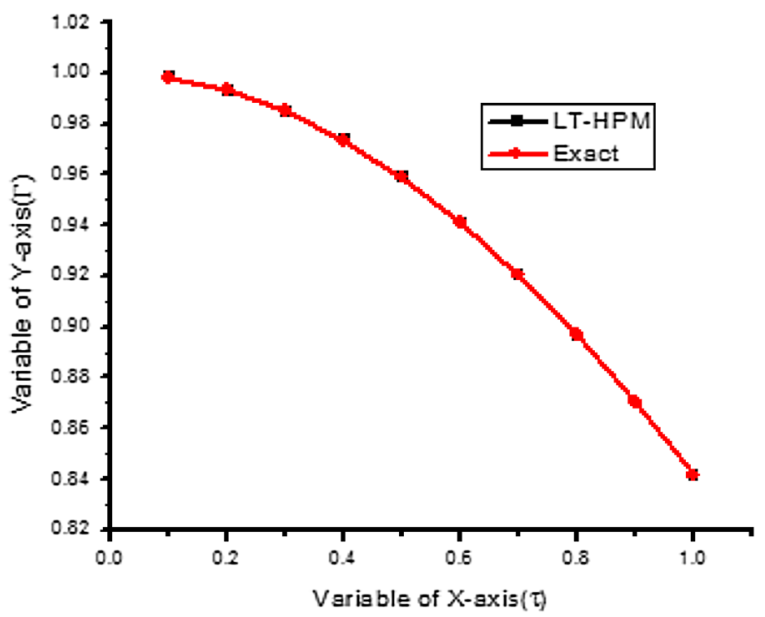

Example 3 Consider the linear, non-homogenous Lane-Emden equation

$$
\Gamma^{\prime \prime}+\frac{2}{\tau} \Gamma^{\prime}+\Gamma=6+12 \tau+\tau^{2}+\tau^{3}, \quad 0 \leq \tau \leq 2,
$$

subject to initial conditions,

$$
\Gamma(0)=0, \quad \Gamma^{\prime}(0)=0 .
$$

Applying Laplace transform on both sides, we get

$$
\begin{aligned}
& L\left(\tau \Gamma^{\prime \prime}\right)+2 L\left(\Gamma^{\prime}\right)+L(\tau \Gamma)=L\left(6 \tau+12 \tau^{2}+\tau^{3}+\tau^{4}\right), \\
& L^{\prime}(\Gamma)=s^{-2} L(\tau \Gamma)-s^{-2} L\left\{6 \tau+12 \tau^{2}+\tau^{3}+\tau^{4}\right\} .
\end{aligned}
$$

By integrating above equation with respect to $s$, we get

$$
L(\Gamma)=-\int s^{-2} L\left(6 \tau+12 \tau^{2}+\tau^{3}+\tau^{4}\right) d s+\int s^{-2} L(\tau \Gamma) d s .
$$

Applying Inverse Laplace transform on both sides, we get

$$
\Gamma(\tau)=-L^{-1}\left(\int-s^{-2} L\left(6 \tau+12 \tau^{2}+\tau^{3}+\tau^{4}\right) d s\right)+L^{-1}\left(\int s^{-2} L(\tau \Gamma) d s\right) .
$$

Applying Homotopy Perturbation Method, we get

$$
\sum_{n=0}^{\infty} p^{n} \Gamma_{n}=\tau^{2}+\tau^{3}+\frac{\tau^{4}}{20}+\frac{\tau^{5}}{30}+p L^{-1}\left(\int s^{-2} L\left(\sum_{n=0}^{\infty} p^{n} \Gamma_{n}(\tau)\right) d s\right) .
$$

equating the coefficient of like power of $\mathrm{p}$, we get 


$$
\begin{aligned}
& p^{0}: \Gamma_{0}=\tau^{2}+\tau^{3}+\frac{\tau^{4}}{20}+\frac{\tau^{5}}{30}, \\
& p^{1}: \Gamma_{1}=L^{-1}\left(\int s^{-2}\left(L\left(\tau \Gamma_{0}(\tau)\right)\right) d s\right)=-\frac{\tau^{4}}{20}-\frac{\tau^{5}}{30}-\frac{\tau^{6}}{840}-\frac{\tau^{7}}{1680}, \\
& p^{2}: \Gamma_{2}=L^{-1}\left(\int s^{-2}\left(L\left(\tau \Gamma_{1}(\tau)\right)\right) d s\right)=\frac{\tau^{6}}{840}+\frac{\tau^{7}}{1680}+\frac{\tau^{8}}{60480}+\frac{\tau^{9}}{151200}, \\
& p^{3}: \Gamma_{3}=L^{-1}\left(\int s^{-2}\left(L\left(\tau \Gamma_{2}(\tau)\right)\right) d s\right)=-\frac{\tau^{8}}{60480}-\frac{\tau^{9}}{151200}-\frac{\tau^{10}}{6652800}-\frac{\tau^{11}}{19958400}, \\
& p^{4}: \Gamma_{4}=L^{-1}\left(\int\left(L\left(\tau \Gamma_{3}(\tau)\right)\right) d s\right)=\frac{\tau^{10}}{6652800}+\frac{\tau^{11}}{19958400}+\frac{\tau^{12}}{1037836800}+\frac{\tau^{13}}{363235600},
\end{aligned}
$$

Thus we get the solution of this series as follows:

$$
\begin{aligned}
& \Gamma=\Gamma_{0}+\Gamma_{1}+\Gamma_{2}+\Gamma_{3}+\Gamma_{4}+\cdots . \\
& \Gamma=\tau^{2}+\tau^{3} .
\end{aligned}
$$

The closed form of the series (36) is $\Gamma(\tau)=\tau^{2}+\tau^{3}$ which gives an exact solution of the problem.

The comparison with exact solution is given by Table 3 .

The Table 3 shows the comparison of values of LT-HPM with exact in the terms of different values of $\tau$.

Example 4 Consider the non-linear, homogenous Lane-Emden equation

$$
\Gamma^{\prime \prime}+\frac{2}{\tau} \Gamma^{\prime}+\Gamma^{3}-\left(6+\tau^{6}\right)=0 \quad \tau \geq 0,
$$

subject to the initial condition

$$
\Gamma(0)=0, \quad \Gamma^{\prime}(0)=0 .
$$

Applying Laplace transform on both sides, we

$$
\begin{aligned}
& L\left(\tau \Gamma^{\prime \prime}\right)+2 L\left(\Gamma^{\prime}\right)+L\left(\tau \Gamma^{3}\right)-L\left(6 \tau+\tau^{7}\right)=0, \\
& \quad-s^{2} L^{\prime}(\Gamma)=L\left(6 \tau+\tau^{7}\right)-L\left(\tau \Gamma^{3}\right), \\
& L^{\prime}(\Gamma)=-s^{-2} L\left(6 \tau+\tau^{7}\right)+s^{-2} L\left(\tau \Gamma^{3}\right), \\
& L(\Gamma(\tau))=-\int s^{-2} L\left(6 \tau+\tau^{7}\right) d s+\int s^{-2} L\left(\tau \Gamma^{3}\right) d s,
\end{aligned}
$$

\section{Table 3 The comparison with the exact solution}

\begin{tabular}{lll}
\hline $\boldsymbol{\tau}$ & LT-HPM & Exact \\
\hline 0.0 & 0.0 & 0.0 \\
0.1 & 0.011 & 0.011 \\
0.2 & 0.048 & 0.048 \\
0.3 & 0.117 & 0.117 \\
0.4 & 0.224 & 0.224 \\
0.5 & 0.375 & 0.375 \\
0.6 & 0.576 & 0.576 \\
0.7 & 0.833 & 0.833 \\
0.8 & 1.152 & 1.152 \\
0.9 & 1.539 & 1.539 \\
1.0 & 2 & 2 \\
\hline
\end{tabular}


Applying Inverse Laplace Transformation, we get

$$
\Gamma(\tau)=-L^{-1}\left(\int s^{-2}\left(L\left(\tau \Gamma^{3}\right)\right) d s\right)+L^{-1}\left(\int s^{-2} L\left(6 \tau+\tau^{7}\right) d s\right) .
$$

Applying HPM both side, we get

$$
\sum_{n=0}^{\infty} p^{n} \Gamma_{n}(\tau)=\tau^{2}+\frac{\tau^{8}}{72}+p L^{-1}\left(\int s^{-2} L\left(\sum_{n=0}^{\infty} p^{n} H_{n}(\Gamma)\right)\right) .
$$

Equating the coefficient of like power of $\mathrm{p}$, we get

$$
\begin{aligned}
p^{0}: \Gamma_{0}(\tau)= & \tau^{2}+\frac{\tau^{8}}{72}, \\
p^{1}: \Gamma_{1}(\tau)= & \int s^{-2} L\left(\tau H_{0}(\Gamma)\right)=-\frac{\tau^{8}}{72}-\frac{\tau^{14}}{5040}-\frac{\tau^{20}}{725760}-\frac{\tau^{26}}{262020096} \\
p^{2}: \Gamma_{2}(\tau)= & \int s^{-2} L\left(\tau H_{1}(\Gamma)\right)=\frac{\tau^{14}}{5040}+\frac{53 \tau^{20}}{12700800}+\frac{25 \tau^{26}}{611380224}+\frac{67 \tau^{32}}{293462507520} \\
& +\frac{491 \tau^{38}}{652367154216960}-\frac{\tau^{44}}{896486037258240} \\
p^{3}: \Gamma_{3}(\tau)= & \int s^{-2} L\left(\tau H_{2}(\Gamma)\right)=-\frac{71 \tau^{20}}{25401600}-\frac{8173 \tau^{26}}{106991539200}-\frac{37369 \tau^{32}}{37661021798400} \\
& -\frac{72109 \tau^{38}}{9133140159037440}-\frac{4514651 \tau^{44}}{108501705089364787200} \\
& -\frac{52841339 \tau^{50}}{368905797303840276480000}-\frac{276053 \tau^{56}}{1068698394448207408005120} \\
& +\frac{\tau^{62}}{101136362276346837073920}, \\
+ & \frac{298992611 \tau^{74}}{397496390291087651691073162444800}, \\
p^{4}: \Gamma_{4}(\tau)= & \int s^{-2} L\left(\tau H_{3}(\Gamma)\right)=\frac{12619 \tau^{26}}{320974617600}+\frac{49591 \tau^{32}}{37661021798400}+\frac{124658383 \tau^{38}}{5860431602049024000} \\
& +\frac{11319804851 \tau^{44}}{52216445574256803840000}-\frac{19811411311 \tau^{50}}{12911702905634409676800000} \\
+ & \frac{575925932801 \tau^{56}}{74185480214613064239022080000} \\
& \frac{3395344051061 \tau^{68}}{96214318223818043730058301931520000} \\
& \frac{636488953519 \tau^{70}}{5632426047399250357186524610560000} \\
& \\
&
\end{aligned}
$$

Thus we get the solution of this series as follows

$$
\begin{aligned}
& \Gamma=\Gamma_{0}+\Gamma_{1}+\Gamma_{2}+\Gamma_{3}+\Gamma_{4}+\cdots . \\
& \Gamma=\tau^{2} .
\end{aligned}
$$


The closed form of the series (40), is $\Gamma(\tau)=\tau^{2}$ which gives an exact solution of the problem.

The comparison with exact solution is given by Table 4 .

The Table 4 shows the comparison of values of LT-HPM with exact in the terms of different values of $\tau$.

Example 5 Consider the linear homogeneous differential equation

$$
\Gamma^{\prime \prime}+\frac{2}{\tau} \Gamma^{\prime}+e^{\Gamma}=0,
$$

with the initial conditions

$$
\Gamma(0)=0, \quad \Gamma^{\prime}(0)=0 .
$$

Applying the Laplace transform on both sides, we get

$$
\begin{aligned}
& L\left(\tau \Gamma^{\prime \prime}\right)+2 \Gamma^{\prime}+L\left(\tau e^{\Gamma}\right)=0, \\
& \quad-s^{2} L^{\prime}(\Gamma)=L\left(\tau e^{\Gamma}\right), \\
& L^{\prime}(\Gamma)=-s^{-2} L\left(\tau e^{\Gamma}\right) .
\end{aligned}
$$

By integrating both sides with respect to s, we get

$$
L(\Gamma)=-\int s^{-2} L\left(\tau e^{\Gamma}\right) d s .
$$

Applying inverse Laplace Transformation on both sides, we get

$$
\Gamma(\tau)=-L^{-1} \int s^{-2}\left(L\left(\tau e^{\Gamma}\right)\right) d s .
$$

Applying HPM on both sides, we get

$$
\sum_{n=0}^{\infty} p^{n} \Gamma_{n}(\tau)=-p L^{-1}\left(\left(\sum_{n=0}^{\infty} \int s^{-2}\left(L\left(\tau e^{\Gamma}\right)\right)\right)\right) d s .
$$

\section{Table 4 The comparison with exact solution}

\begin{tabular}{lll}
\hline $\boldsymbol{\tau}$ & LT-HPM & Exact \\
\hline 0.0 & 0.0 & 0.0 \\
0.1 & 0.1 & 0.1 \\
0.2 & 0.4 & 0.4 \\
0.3 & 0.9 & 0.9 \\
0.4 & 0.16 & 0.16 \\
0.5 & 0.25 & 0.25 \\
0.6 & 0.36 & 0.36 \\
0.7 & 0.49 & 0.49 \\
0.8 & 0.64 & 0.64 \\
0.9 & 0.81 & 0.81 \\
1.0 & 1.0 & 1.0 \\
\hline
\end{tabular}


Equating the coefficient of like power of $\mathrm{p}$, we get

$$
\begin{aligned}
& p^{0}: \Gamma_{0}(\tau)=0, \\
& p^{1}: \Gamma_{1}(\tau)=-\frac{\tau^{2}}{3 !}, \\
& p^{2}: \Gamma_{2}(\tau)=\frac{\tau^{4}}{5 !}, \\
& p^{3}: \Gamma_{3}(\tau)=-\frac{\tau^{6}}{7 !}, \\
& p^{4}: \Gamma_{4}(\tau)=\frac{\tau^{8}}{9 !},
\end{aligned}
$$

Thus we get the solution of this series as follows

$$
\begin{aligned}
\Gamma & =\Gamma_{0}+\Gamma_{1}+\Gamma_{2}+\Gamma_{3}+\Gamma_{4}+\cdots . \\
& =-\frac{\tau^{2}}{3 !}+\frac{\tau^{4}}{5 !}-\frac{\tau^{6}}{7 !}+\frac{\tau^{8}}{9 !}-\cdots .
\end{aligned}
$$

Example 6 Consider the nonlinear, homogeneous Lane-Emden differential equation

$$
\Gamma^{\prime \prime}+\frac{2}{\tau} \Gamma^{\prime}+4\left(2 e^{(\Gamma)}+e^{\left(\frac{\Gamma}{2}\right)}\right)=0, \quad 0 \leq \tau \leq 1,
$$

subject to initial conditions,

$$
\Gamma(0)=0, \quad \Gamma^{\prime}(0)=0 .
$$

The exact solution of the problem is

$$
\Gamma(\tau)=-2 \operatorname{In}\left(1+\tau^{2}\right) .
$$

Applying Laplace transform on both sides, we get

$$
\begin{aligned}
& L\left(\tau \Gamma^{\prime \prime}\right)+2 L\left(\Gamma^{\prime}\right)+L\left(4\left(2 \tau e^{(\Gamma)}+\tau e^{\left(\frac{\Gamma}{2}\right)}\right)\right)=0, \\
& -s^{2} L^{\prime}(\Gamma)+4 L\left(2 \tau e^{(\Gamma)}+\tau e^{\left(\frac{\Gamma}{2}\right)}\right)=0, \\
& L^{\prime}(\Gamma)=s^{-2} 4\left(L\left(2 \tau e^{(\Gamma)}+\tau e^{\left(\frac{\Gamma}{2}\right)}\right)\right) .
\end{aligned}
$$

Integrating the above equation with respect to $\mathrm{s}$, we get

$$
\Gamma(s)=\int s^{-2} L\left(4\left(2 \tau e^{(\Gamma)}+\tau e^{\left(\frac{\Gamma}{2}\right)}\right)\right) d s .
$$

Applying Inverse Laplace Transformation on both sides, we get

$$
\Gamma=L^{-1}\left(\int s^{-2}\left(L\left(4 \tau \Gamma^{3}\right)\right) d s\right) .
$$


Applying HPM on both sides, we get

$$
\sum_{n=0}^{\infty} p^{n} \Gamma_{n}(\tau)=p L^{-1}\left(\int s^{-2} L\left(\sum_{n=0}^{\infty} p^{n}\left(4 \tau H_{n}(\Gamma)\right) d s\right)\right) .
$$

where $N(\Gamma)=\left(2 e^{(\Gamma)}+e^{\left(\frac{\Gamma}{2}\right)}\right)$ is the nonlinear operator, $H_{n}(\Gamma)$ is the He's polynomial. Which is given by

$$
\left.\begin{array}{l}
H_{0}(\Gamma)=\left(2 e^{\left(\Gamma_{0}\right)}+e^{\left(\frac{\Gamma_{0}}{2}\right)}\right), \\
H_{1}(\Gamma)=\Gamma_{1}\left(2 e^{\left(\Gamma_{1}\right)}+\frac{1}{2} e^{\left(\frac{\Gamma_{1}}{2}\right)}\right), \\
H_{2}(\Gamma)=\Gamma_{2}\left(2 e^{\left(\Gamma_{1}\right)}+\frac{1}{2} e^{\left(\frac{\Gamma_{1}}{2}\right)}\right)+\frac{\Gamma_{1}^{2}}{2 !}\left(2 e^{\left(\Gamma_{1}\right)}+\frac{1}{4} e^{\left(\frac{\Gamma_{1}}{2}\right)}\right),
\end{array}\right\}
$$

using the value of Eq. (50) in Eq. (49) and equating the coefficient of like the power of p, we get

$$
\begin{aligned}
& \left.\begin{array}{l}
p^{0}: \Gamma_{0}=0, \\
p^{1}: \Gamma_{1}=\left(\int s^{-2}\left(L\left(4 \tau\left(H_{0}\right)\right)\right) d s\right)=-2 \tau^{2}, \\
p^{2}: \Gamma_{2}=\left(\int s^{-2}\left(L\left(4 \tau\left(H_{1}\right)\right)\right) d s\right)=\tau^{4},
\end{array}\right\} \\
& \left.\begin{array}{l}
p^{3}: \Gamma_{3}=\left(\int s^{-2}\left(L\left(4 \tau\left(H_{2}\right)\right)\right) d s\right)=-\frac{2}{3} \tau^{6}, \\
p^{4}: \Gamma_{4}=\left(\int s^{-2}\left(L\left(4 \tau\left(H_{3}\right)\right)\right) d s\right)=\frac{1}{2} \tau^{8}, \\
\vdots
\end{array}\right\}
\end{aligned}
$$

Thus we get the solution of this series as follows:

$$
\begin{gathered}
\Gamma=\Gamma_{0}+\Gamma_{1}+\Gamma_{2}+\Gamma_{3}+\Gamma_{4}+\cdots \\
\Gamma(\tau)=-2\left(\tau^{2}-\frac{1}{2} \tau^{4}+\frac{1}{3} \tau^{6}-\frac{1}{4} \tau^{8}+\cdots\right)
\end{gathered}
$$

The closed form of the series (52) is $\Gamma(\tau)=-2 \operatorname{In}\left(1+\tau^{2}\right)$ which gives an exact solution of the problem.

Example 7 Consider the nonlinear, homogeneous Lane-Emden differential equation

$$
\Gamma^{\prime \prime}+\frac{2}{\tau} \Gamma^{\prime}-6 \Gamma=4 \Gamma \operatorname{In} \Gamma, \quad 0 \leq \tau \leq 1,
$$

subject to the initial condition,

$$
\Gamma(0)=1, \quad \Gamma(0)=0 .
$$


The exact solution of the equation is

$$
\Gamma(\tau)=e^{\tau^{2}} .
$$

Applying Laplace Transform on both sides, we get

$$
\begin{aligned}
& L\left(\tau \Gamma^{\prime \prime}\right)+L\left(2 \Gamma^{\prime}\right)-L(6 \tau \Gamma)-L(4 \tau(\Gamma \operatorname{In} \Gamma))=0, \\
& -s^{2} L^{\prime}(\Gamma)-1=L\{6 \tau \Gamma+4 \Gamma \tau \operatorname{In} \Gamma\}, \\
& L^{\prime}(\Gamma)=-\frac{1}{s^{2}}-\frac{1}{s^{2}} L\{6 \tau \Gamma+4 \Gamma \tau \operatorname{In} \Gamma\} .
\end{aligned}
$$

Taking integration on both sides of above equation, we get

$$
L(\Gamma)=-\int s^{-2} d s-\int s^{-2} L\{6 \tau \Gamma+4 \Gamma \tau \operatorname{In} \Gamma\} d s .
$$

Applying Inverse Laplace transform on both sides, we get

$$
\Gamma(\tau)=1-L^{-1}\left(\int s^{-2} L(6 \tau \Gamma+4 \Gamma \tau \operatorname{In} \Gamma) d s\right) .
$$

Applying HPM on both sides, we get

$$
\sum_{n=0}^{\infty} p^{n} \Gamma_{n}(\tau)=1-p L^{-1}\left(\int s^{-2} L\left(\sum_{n=0}^{\infty} p^{n} \Gamma_{n}(\tau)\right) d s\right) .
$$

Equating the coefficient of like power of $p$, we get

$$
\begin{aligned}
& p^{0}: \Gamma_{0}(\tau)=1 \\
& p^{1}: \Gamma_{1}(\tau)=-\int s^{-2} L\left(6 \tau \Gamma_{0}+4 \Gamma_{0}\left(\tau \operatorname{In} \Gamma_{0}\right)\right) d s=\tau^{2}, \\
& p^{2}: \Gamma_{2}(\tau)=-\int s^{-2} L\left(6 \tau \Gamma_{1}+4 \Gamma_{1}\left(\tau \operatorname{In} \Gamma_{1}\right)\right) d s=\frac{1}{2 !} \tau^{4}, \\
& p^{3}: \Gamma_{3}(\tau)=-\int s^{-2} L\left(6 \tau \Gamma_{2}+4 \Gamma_{2}\left(\tau \operatorname{In} \Gamma_{2}\right)\right) d s=\frac{1}{3 !} \tau^{6}, \\
& p^{4}: \Gamma_{4}(\tau)=-\int s^{-2} L\left(6 \tau \Gamma_{3}+4 \Gamma_{3}\left(\tau \operatorname{In} \Gamma_{3}\right)\right) d s=\frac{1}{4 !} \tau^{8}, \\
& p^{5}: \Gamma_{5}(\tau)=-\int s^{-2} L\left(6 \tau \Gamma_{4}+4 \Gamma_{4}\left(\tau \operatorname{In} \Gamma_{4}\right)\right) d s=\frac{1}{5 !} \tau^{10},
\end{aligned}
$$

Thus we get the solution of this series as follows:

$$
\begin{aligned}
& \Gamma=\Gamma_{0}+\Gamma_{1}+\Gamma_{2}+\Gamma_{3}+\Gamma_{4}+\cdots \cdots \\
& \quad=1+\tau^{2}+\frac{1}{2 !} \tau^{4}+\frac{1}{3 !} \tau^{6}+\frac{1}{4 !} \tau^{8}+\frac{1}{5 !} \tau^{10}+\cdots . \\
& \Gamma(\tau)=\exp \left(\tau^{2}\right) .
\end{aligned}
$$


The closed form of the series (57) is $\Gamma(\tau)=\exp \left(\tau^{2}\right)$ which gives an exact solution of the problem.

The Table 5 shows the comparison of values of LT-HPM with exact in the terms of different values of $\tau$.

Example 8 Consider the following Lane-Emden type differential equation:

$$
\Gamma^{\prime \prime}+\frac{2}{\tau} \Gamma^{\prime}+\sin (\Gamma)=0,
$$

subject to the initial condition

$$
\Gamma(0)=1, \quad \Gamma^{\prime}(0)=0 .
$$

Applying Laplace Transform (LT) on both sides, we get

$$
\begin{gathered}
L\left(\tau \Gamma^{\prime \prime}\right)+2 \Gamma^{\prime}+L(\tau \sin (\Gamma))=0, \\
-s^{2} L^{\prime}(\Gamma)-1=-L(\tau \sin (\Gamma)), \\
L^{\prime}(\Gamma)=-\frac{1}{s^{2}}+\frac{1}{s^{2}}(L(\tau \sin (\Gamma))),
\end{gathered}
$$

Integrating the above equation with respect to $s$, we get

$$
\Gamma(s)=-\int s^{-2} d s+\int s^{-2}((L(\tau \sin (\Gamma)))) d s .
$$

Applying ILT on both sides, we get

$$
\Gamma(\tau)=1+L^{-1}\left(\int s^{-2}((L(\tau \sin (\Gamma)))) d s\right) .
$$

Applying HPM on both sides, we get

$$
\sum_{n=0}^{\infty} p^{n} \Gamma_{n}(\tau)=1-p L^{-1}\left(\int s^{-2}\left(\sum_{n=0}^{\infty}\left(L\left(\tau H_{n}(\Gamma)\right)\right)\right) d s\right) .
$$

Here $\zeta(\Gamma)=\sin (\Gamma)$ is a non-linear term and $H_{n}(\Gamma)$ is He's polynomial.

\section{Table 5 The comparison with exact solution}

\begin{tabular}{llll}
\hline $\boldsymbol{\tau}$ & LT-HPM & Exact & Error \\
\hline 0.0 & 1 & 1 & 0.0 \\
0.1 & 1.010050167 & 1.010050167 & 0.0 \\
0.2 & 1.040810773 & 1.040810774 & $1 \mathrm{E}-9$ \\
0.3 & 1.094174234 & 1.094174284 & $5 \mathrm{E}-8$ \\
0.4 & 1.173509973 & 1.173510871 & $8.98 \mathrm{E}-7$ \\
0.5 & 1.284016927 & 1.284025417 & $8.49 \mathrm{E}-6$ \\
0.6 & 1.43327584 & 1.433329415 & 0.19904038 \\
0.7 & 1.632060167 & 1.63231622 & $2.56053 \mathrm{E}-4$ \\
0.8 & 1.895481173 & 1.896480879 & $9.99706 \mathrm{E}-4$ \\
0.9 & 2.359156039 & 2.247907987 & -0.111248052 \\
1.0 & 2.708333333 & 2.718281828 & 0.009948495 \\
\hline
\end{tabular}




$$
\begin{aligned}
& H_{0}=\sin \Gamma_{0}, \\
& H_{1}=\Gamma_{1} \cos \Gamma_{0}, \\
& H_{2}=-\left(\frac{\Gamma_{1}^{2}}{2}\right) \sin \Gamma_{0}+\Gamma_{2} \cos \Gamma_{0}, \\
& H_{3}=-\left(\frac{\Gamma_{1}^{3}}{6}\right) \cos \Gamma_{0}-\Gamma_{1} \Gamma_{2} \sin \Gamma_{0}+\Gamma_{3} \cos \Gamma_{0},
\end{aligned}
$$

Equating the coefficients like power of $\mathrm{p}$, we get

$$
\begin{aligned}
& p^{0}: \Gamma_{0}=1, \\
& p^{1}: \Gamma_{1}=L^{-1}\left\{\int s^{-2} L\left(\tau H_{0}\right) d s\right\}=-\frac{\tau^{2}}{6} k_{1}, \\
& p^{2}: \Gamma_{2}=L^{-1}\left\{\int s^{-2} L\left(\tau H_{1}\right) d s\right\}=\frac{1}{120} k_{1} k_{2} \tau^{4}, \\
& p^{3}: \Gamma_{3}=L^{-1}\left\{\int s^{-2} L\left(\tau H_{2}\right) d s\right\}=k_{1}\left(\frac{1}{3024} k_{1}^{2}-\frac{1}{5040} k_{1} k_{2}^{2}\right) \tau^{6}, \\
& p^{4}: \Gamma_{4}=L^{-1}\left\{\int s^{-2} L\left(\tau H_{3}\right) d s\right\}=k_{1} k_{2}\left(\frac{-107}{3265920} k_{1}^{2}+\frac{1}{362880} k_{2}^{2}\right) x^{8},
\end{aligned}
$$

Thus we get the solution of this series as follows:

$$
\begin{aligned}
\Gamma= & \Gamma_{0}+\Gamma_{1}+\Gamma_{2}+\Gamma_{3}+\Gamma_{4}+\cdots \\
\Gamma= & 1-\frac{\tau^{2}}{6} k_{1}+\frac{1}{120} k_{1} k_{2} \tau^{4}+k_{1}\left(\frac{1}{3024} k_{1}^{2}-\frac{1}{5040} k_{1} k_{2}^{2}\right) \tau^{6} \\
& +k_{1} k_{2}\left(\frac{-107}{3265920} k_{1}^{2}+\frac{1}{362880} k_{2}^{2}\right) x^{8}+\cdots(61)
\end{aligned}
$$

where

$$
k_{1}=\sin (1), \quad k_{2}=\cos (1),
$$

\section{Conclusions}

In this communication, we have successfully employed the Homotopy Perturbation Method with Laplace Transform (LT-HPM) to obtain exact solutions for singular IVPs of Lane-Emden-type equations. We also find the accuracy of this method which gives us very attractive results in the terms of power series. This method can accelerate the rapid convergence of series solution when compared with Homotopy Perturbation Method using Laplace Transform. Very recently, the LT-HPM has been extensively applicable in many fields of science and engineering to solve these types of problems because of its dependability and the attenuation in the size of computations. The graphical representation of such types of problems shows that the LT-HPM is a promising tool for singular IVP's of Lane-Emden type, and in some cases, yields exact solutions in two iterations. 


\section{Authors' contributions}

Ms. RT drafted the manuscript. Dr. HKM made some revisions of the manuscript. Both authors have read and approved the final manuscript.

\section{Acknowledgements}

The second author acknowledges the financial support provided by the Madhya Pradesh Council of Science and Technology (MPCST), under research Grant No. 1013/CST/R\&D/Phy\&EnggSc/2015; Bhopal, Madhya Pradesh, India. The authors also extended their appreciations to anonymous reviewers for their valuable suggestions to revise this paper.

\section{Competing interests}

The authors declare they have no competing interests.

Received: 19 May 2016 Accepted: 6 October 2016

Published online: 22 October 2016

\section{References}

Abbasbandy S (2006) Application of He's Homotopy Perturbation Method for Laplace Transform. Chaos Solitons Fractals 30(5):1206-1212

Baranwal VK, Pandey RK, Tripathi MP, Singh OP (2012) An analytic algorithm of Lane-Emden-type equation arising in astrophysics-a hybrid approach. J Theor Appl Phys 6(22):1-16

Chandrasekhar S (1967) Introduction to the study of stellar structure. Dover, New York

Chowdhury MSH, Hashim I (2007) Solutions of a class of singular second-order ivps by homotopy perturbation method. Phys Lett A 365(5-6):439-447

Cveticanin L (2006) Homotopy-perturbation method for pure nonlinear differential equation. Chaos, Solitons Fractals 30:1221-1230

Davis HT (1962) Introduction to nonlinear differential and integral equations. Dover, New York

Dixon JM, Tuszynski JA (1990) Solutions of a generalized Emden equation and their physical significance. Phys Rev A 41:4166-4173

Fermi E (1927) Un metodo statistico per la determinazione di alcune priorieta dell atome. Rend Accad Naz Lincei 6:602-607

Fowler RH (1930) The solutions of Emden's and similar differential equations. MNRAS 91:63-91

Frank-Kamenetskii DA (1921) Diffusion and heat exchange in chemical kinetics. Princeton University Press, Princeton, 1955

Ganji DD, Rajabi A (2006) Assessment of homotopy-perturbation and perturbation methods in heat radiation equations. Int Commun Heat Mass Transfer 33:391-400

Goenner H, Havas P (2000) Exact solutions of the generalized Lane-Emden equation. Math Phys 41(10):7029-7042

Gupta VG, Gupta S (2011) Homotopy perturbation transform method for solving initial boundary value problems of variable coefficients. Int J Nonlinear Sci 12(3):270-277

Gupta S, Kumar D, Singh J (2015) Analytical solutions of convection-diffusion problems by combining Laplace transform method and homotopy perturbation method. Alexendria Eng J 54(3):645-651

Hashim I, Chowdhury MSH (2007) Adaptation of homotopy-perturbation method for the numeric-analytic solution of system of odes. Phys Lett A 4(21):470-481

He JH (1999a) Homotopy perturbation technique. Comput Methods Appl Mech Eng 178:257-262

He JH (1999b) Homotopy perturbation technique. Comput Methods Appl Mech Eng 178(3-4):257-262

He JH (2003a) Homotopy perturbation method a new nonlinear analytic technique. Appl Math Comput 135(1):73-79

He JH (2003b) Variational approach to the Lane-Emden equation. Appl Math Comput 143:539-541

He JH (2006) New interpretation of homotopy perturbation method. Int J Mod Phys B 20(18):2561-2568

Hosseini SG, Abbasbandy S (2015) Solution of Lane-Emden type equations by combination of the spectral method and adomian decomposition method. Hindawi Publ Corp Math Probl Eng 2015:1-10

Jang TS (2016) A new solution procedure for a nonlinear infinite beam equation of motion. Commun Nonlinear Sci Numer Simul 2016(39):321-331

Jang TS (2017) A new dispersion-relation preserving method for integrating the classical Boussinesq equation. Commun Nonlinear Sci Numer Simul 43:118-138

Jiwari R, Mittal RC (2011) A higher order numerical scheme for singularly perturbed Burger-Huxley Equation. J Appl Math Informatics 29(No. 3-4):813-829

Khuri SA, Sayfy A (2012) A Laplace variational iteration strategy for the solution of differential equations. Appl Math Lett 25(12):2298-2305

Lane JH (1870) On the theoretical temperature of the sun under the hypothesis of a gaseous mass maintaining its volume by its internal heat and depending on the laws of gases known to terrestrial experiment. Am J Sci Arts 2(50):57-74

Liao S (2003) A new analytic algorithm of Lane-Emden type equations. Appl Math Comput 142(1):1-16

Madani M, Fathizadeh M, Khan Y, Yildirim A (2011) On the coupling of the homotopy perturbation method and Laplace Transformation. Math Comput Model 53(9-10):1937-1945

Mandelzweig VB, Quasi TF (2001) Linearization approach to nonlinear problems in physics with application to nonlinear odes. Comput Phys Commun 141:268-281

Merdan M, Khaniyev T (2010) Homotopy perturbation method for solving the viral dynamical model, C.Ü. Fen-Edebiyat Fakültesi Fen Bilimleri Dergisi 31(1):65-77 
Mishra HK, Nagar AK (2012) He-Laplace method for linear and nonlinear partial differential equations. J Appl Math 2012:1-16

Mishra HK (2014) He-Laplace Method for the solution of two-point boundary value problems. Am J Math Anal 2(3):45-49

Momani S, Erjaee GH, Alnasr MH (2009) The modified homotopy perturbation method for solving strongly nonlinear oscillators. Comput Math Appl 58:2209-2220

Motsa SS, Sibanda P (2010) A new algorithm for singular IVPs of Lane-Emden type. Latest Trends Appl Math Simul Model 210(3):176-180

Nazari-Golshan A, Nourazar SS, Ghafoori-Fard H, Yildirim A, Campo A (2013) A modified homotopy perturbation method coupled with the Fourier transform for nonlinear and singular Lane-Emden equations. Appl Math Lett 26(10):1018-1025

Nouh MI (2004) Accelerated power series solution of polytropic and isothermal gas spheres. New Astron 9:467-473

Pandit S (2014) Haar wavelet approach for numerical solution of two parameters singularly perturbed boundary value problems. Appl Math Inf Sci 6:2965-2974

Rafei M, Ganji DD (2006) Explicit solutions of Helmholtz equation and fifth-order KdV equation using homotopy perturbation method. Int J Nonlinear Sci Numer Simul 7(3):321-328

Rafiq A, Hussain S, Ahmed M (2009) General homotopy method for Lane-Emden type differential equations. Int J Appl Math Mech 5(3):75-83

Ramos Jl (2003) Linearization methods in classical and quantum mechanics. Comput Phys Commun 153(2):199-208

Ramos JI (2008) Series approach to the Lane-Emden equation and comparison with the homotopy perturbation method. Chaos, Solitons Fractals 38(2):400-408

Richardson OW (1921) The emission of electricity from hot bodies. Longmans Green and Co., London

Shakeri F, Dehghan M (2007) Inverse problem of diffusion equation by he's homotopy perturbation method. Phys Scr 75(4):551-556

Sharma D (2012) A comparative study of model of matrix and finite elements methods for two-point boundary value problems. Int J Appl Math Mech 8(16):29-45

Sharma D, Jiwari R, Kumar S (2012) Numerical solution of two-point boundary value problems using Galerkin-Finite element method. Int J Nonlinear Sci 13(2):204-210

Shawagfeh NT (1993) Nonperturbative approximate solution for Lane-Emden equation. J Math Phys 34(9):4364

Singh J, Kumar D, Rathore S (2012) Application of homotopy perturbation transform method for solving linear and nonlinear Klein-Gordon equations. J Inf Comput Sci 7(2):131-139

Spiegel MR, Teoríay (1988) Problemas de Transformadas de Laplace, Primeraedición. Serie de compendious Schaum. McGraw-Hill, México

Sweilam NH, Khader MM (2009) Exact solutions of some coupled nonlinear partial differential equations using the homotopy perturbation method. Comput Math Appl 58:2134-2141

Wazwaz AM (2001a) A new algorithm for solving differential equations of Lane-Emden type. Appl Math Comput $118: 287-310$

Wazwaz AM (2001b) A new method for solving singular initial value problems in the second order ordinary differential equations. Appl Math Comput 128:45-57

Wazwaz AM (2011) The variational iteration method for solving nonlinear singular boundary value problems arising in various physical models. Commun Nonlinear Sci Numer Simul 16(10):3881-3886

Yildrim A, Ozis T (2007) Solutions of singular IVPS of Lane-Emden type by homotopy perturbation method. Phys Lett A 369(1-2):70-76

Yin F-K, Han W-Y, Song J-Q (2013) Modified Laplace decomposition method for Lane-Emden type differential equation. Int J Appl Phys Math 3(No. 2): 98-102

\section{Submit your manuscript to a SpringerOpen ${ }^{\circ}$ journal and benefit from:}

- Convenient online submission

- Rigorous peer review

- Immediate publication on acceptance

- Open access: articles freely available online

- High visibility within the field

- Retaining the copyright to your article

Submit your next manuscript at $\boldsymbol{\nabla}$ springeropen.com 